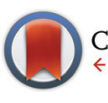

CrossMark $\leftarrow$ click for updates

Cite this: Polym. Chem., 2015, 6 , 2163

Received 2nd December 2014, Accepted 5th January 2015

DOI: $10.1039 / c 4 p y 01665 c$

www.rsc.org/polymers

\section{Thermoresponsive polyelectrolytes derived from ionic liquids}

\begin{abstract}
Yuki Kohno, ${ }^{a, b}$ Shohei Saita, ${ }^{b, c}$ Yongjun Men, ${ }^{d}$ Jiayin Yuan*e and Hiroyuki Ohno*b,c
Ionic liquid (IL)-based polyelectrolytes (PILs), referred to as polymeric ILs, polymerised ILs, or poly(IL)s are a new subclass of polymer materials. They are distinct from conventional polyelectrolytes due to their unique physico-chemical properties originated from a dense packing of ILs in the macromolecular architecture. Mixtures of PILs and solvents, in particular, water have attracted a great deal of interest especially in terms of their compatibilities depending on temperature, namely, thermoresponsiveness of PIL/solvent mixtures. Apart from static compatibility, such as the compatibility of PILs with solvents, which do not change largely by a temperature change, there are mainly two types of dynamic phase changes, an upper critical solution temperature (UCST)- and a lower critical solution temperature (LCST)-type phase behaviour. Some PILs dissolved in solvents homogenise upon heating; this behaviour is classified as UCST behaviour. On the other hand, only in the last two years have PIL/water mixtures with LCST been discovered. This article summarises rapidly growing studies on the design of thermoresponsive PIL systems with water or organic solvents. The hydrophobicity/hydrophilicity balance of the starting IL monomers features the phase behaviour of the resulting polyelectrolytes, and some IL monomers that show thermoresponsive phase behaviour in solvents were found to maintain their thermoresponsiveness even after the polymerisation. Based on their unique combination of properties derived from an ionic and thermoresponsive nature, these thermoresponsive PILs will attract considerable interest, and their wide applications are expected in the fields of separation, sensing and desalination.
\end{abstract}

\section{Introduction}

Functional polymers with thermoresponsive behaviours in various solvents, in particular, water have been widely investigated and considered to be one of the "intelligent" materials. ${ }^{1-9}$ The thermoresponsive phase behaviours of the polymer/solvent mixtures can be subdivided into such two general classes as that with an upper critical solution temperature (UCST) and that with a lower critical solution temperature (LCST) depending on mixing behaviour of the corresponding polymer solutions upon temperature change. Many studies have been carried out on polymers that show the LCST-type phase behaviour in water, in which homogeneously dissolved

\footnotetext{
${ }^{a}$ Department of Chemical \& Biological Engineering, University of Colorado, Boulder, CO80309, USA

${ }^{b}$ Functional Ionic Liquid Laboratories, Graduate School of Engineering, Tokyo University of Agriculture and Technology, Koganei, Tokyo 184-8588, Japan. E-mail: ohnoh@cc.tuat.ac.jp

${ }^{c}$ Department of Biotechnology, Tokyo University of Agriculture and Technology, Nakacho, Koganei, Tokyo 184-8588, Japan

${ }^{d}$ Radboud University Nijmegen, Institute for Molecules and Materials, Heyendaalseweg 135, 6525 AJ, Nijmegen, The Netherlands

${ }^{e}$ Max Planck Institute of Colloids and Interfaces, Research Campus Golm, D-14424 Potsdam, Germany. E-mail: Jiayin.yuan@mpikg.mpg.de
}

polymers are phase-separated from water at elevated temperatures. Poly( $N$-isopropylacrylamide) (PNIPAM) and some nonionic polymers are well-known to show this type of phase behaviour. Various applications of these thermoresponsive polymers have already been developed (e.g. drug delivery, cultured cell detachment, actuator, stabiliser, and absorbent). ${ }^{10-15}$ Although a few partially-charged homopolymers exhibit the LCST-type phase transition with water, ${ }^{16,17}$ there was no example of fully-ionised homopolyelectrolytes that show the LCST-type phase transition in water until recently.

Ionic liquids (ILs) are organic salts that melt at very low temperature (empirically below $\left.100{ }^{\circ} \mathrm{C}\right) .{ }^{18-20}$ Unlike other molecular liquids, ILs have unique properties, such as vanishingly low vapor pressure, high charge density, inflammability and high thermal/chemical stability in the liquid state. As early as 1998, Ohno's group reported a set of polyelectrolytes bearing an IL repeating unit for a new generation of solid electrolytes. $^{21}$ The IL-based polyelectrolytes (hereafter PILs) have been collecting much interest as a distinct subclass of polyelectrolytes, which potentially inherit certain properties derived from ILs (e.g. low glass transition temperatures in spite of their high charge density). ${ }^{22}$ Many studies on PILs so far have been focused on characterising properties of PILs in their neat states for various applications..$^{23,24}$ On the other hand, 
mixtures of PILs with solvents have become a significant research topic recently. Depending on the hydrophobicity/ hydrophilicity balance of component ions of starting IL monomers, which is greatly facilitated by the structural diversity and tremendous cation/anion-choices, a few PILs were found to show LCST- as well as UCST-type phase transition in water.

This review paper aims to summarise recent progress on the design of PILs bearing thermoresponsive phase behaviour with water and other solvents. As several review papers on $\mathrm{ILs}^{18-20}{ }^{18 \mathrm{PIL}},{ }^{23-26}$ and thermoresponsive polymers in $\mathrm{ILs}^{27}$ have already been reported, we recommend to study them to obtain fundamental aspects in this research field. Distinct from these review papers, we focus here exclusively on the phase behaviour of both ILs and PILs with solvents, because of their close relationship in terms of the thermoresponsiveness. The significance of these relationships and potential applications of these thermoresponsive PILs will also be discussed.

\section{Thermoresponsive IL/molecular liquid mixtures}

Prior to describing thermoresponsive PIL systems, it is of great importance to survey IL/solvent systems that show thermoresponsive phase transition in order to overview this field. As mentioned above, there are mainly two distinct thermoresponsive phase behaviours of ILs with solvents; UCST and LCST behaviour. In IL/solvent mixtures showing the UCST behaviour, separated liquid phases are mixed homogeneously upon heating. On the other hand, homogeneous IL solutions become phase-separated upon heating when the mixtures show the LCST-type phase transition. We focus here on both UCST- and LCST-type phase transitions of IL/solvent systems. Fig. 1 shows both structures and abbreviations of all component ions of ILs in this paper. In most cases, the phase tran- sition temperature $\left(T_{\mathrm{c}}\right)$ of IL/solvent mixtures was determined as the temperature at which turbid suspension was generated in the mixture upon changing temperatures. Fig. 2 presents typical phase diagrams depicting the thermoresponsive phase behaviour of IL/solvent mixtures. The $T_{\mathrm{c}}$ values depend on the solvent concentration, and a concave curve is generally observed in a $T_{\mathrm{c}}$-concentration phase diagram that shows the LCST-type phase transition (Fig. 2A). The mixture forms two phases (or one phase) at the temperature range above (or below) the concave curve. In the case of a UCST-type phase transition, a convex curve is seen in the phase diagram (Fig. 2B), and the mixture becomes one phase above the convex curve. Table 1 presents a summary of reported IL/ solvent systems that show thermoresponsive phase transition. In Table 1 , the $T_{\mathrm{c}}$ values of IL/solvent systems are described as those at a critical composition: the molar ratio of solvent molecules to IL at the top (UCST) or bottom (LCST) of the phase diagrams (Fig. 2). The molar ratio at the critical composition has been approximately determined from the reported phase diagrams, and these data are also summarised in Table 1.

The UCST behaviour was commonly observed in ILs after mixing with water or other organic solvents ${ }^{28-38}$ (entries 1-8 as typical examples); this behaviour is reasonably comprehensible from the viewpoint of temperature-solubility relationship of salts in solvents. The UCST behaviour also existed in IL/IL mixtures. ${ }^{39}$ However, the LCST-behaviour was scarcely observed in IL/solvent mixtures until recently, although the LCST-type phase transition itself had been already reported in a few organic salt/water mixtures. In 1989, Weingärtner and co-workers reported an aqueous solution of $\left[\mathrm{N}_{4444}\right] \mathrm{SCN}$ that shows both UCST- and LCST-type phase transition (entry 9). ${ }^{40}$ Although the salt is not classified as an IL by an empirical definition (melting point of the salt is $126.8^{\circ} \mathrm{C}$ ), ${ }^{40}$ to our knowledge, this system is the first example for the LCST-type phase transition of an aqueous salt mixture. A few thermoresponsive

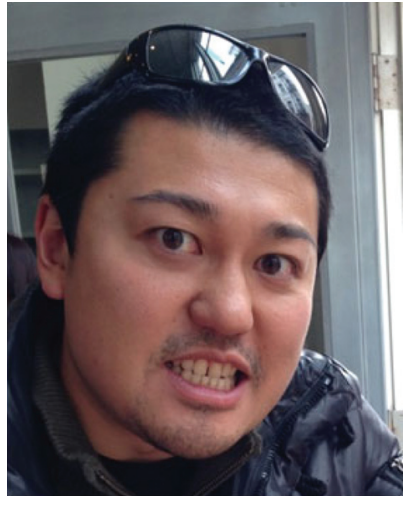

Yuki Kohno
Dr Yuki Kohno received his Ph.D. from Tokyo University of Agriculture and Technology (TUAT), Japan in 2012 under the direction of Prof. Hiroyuki Ohno. After working as a post-doctoral research fellow in TUAT, he joined University of Colorado Boulder (CU), USA in 2013 supervised by Prof. Richard D. Noble and Prof. Douglas L. Gin. He is currently conducting researches in $C U$ under the sponsorship of Japan Society for

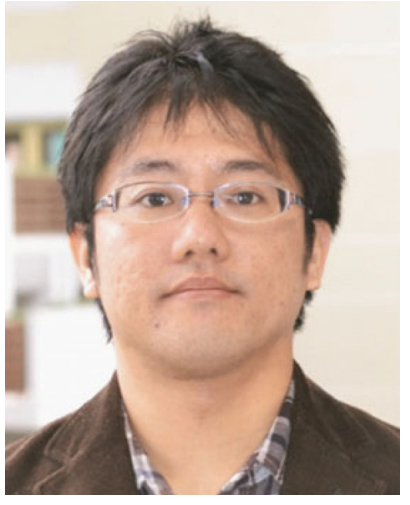

Shohei Saita
Shohei Saita received his BEng (2010) and MEng (2012) degrees from Tokyo University of Agriculture and Technology (TUAT; Japan). He is currently a Ph.D. candidate in the Department of Biotechnology in TUAT under the direction of Prof. H. Ohno. His research is focused on design and control of thermoresponsive phase transition of ionic liquid/ water mixtures.

the Promotion of Science (JSPS Postdoctoral Fellowships for Research Abroad). His current research focus is the design of ionic liquid-based liquid, polymer, and liquid-crystalline materials for gas separation applications. 


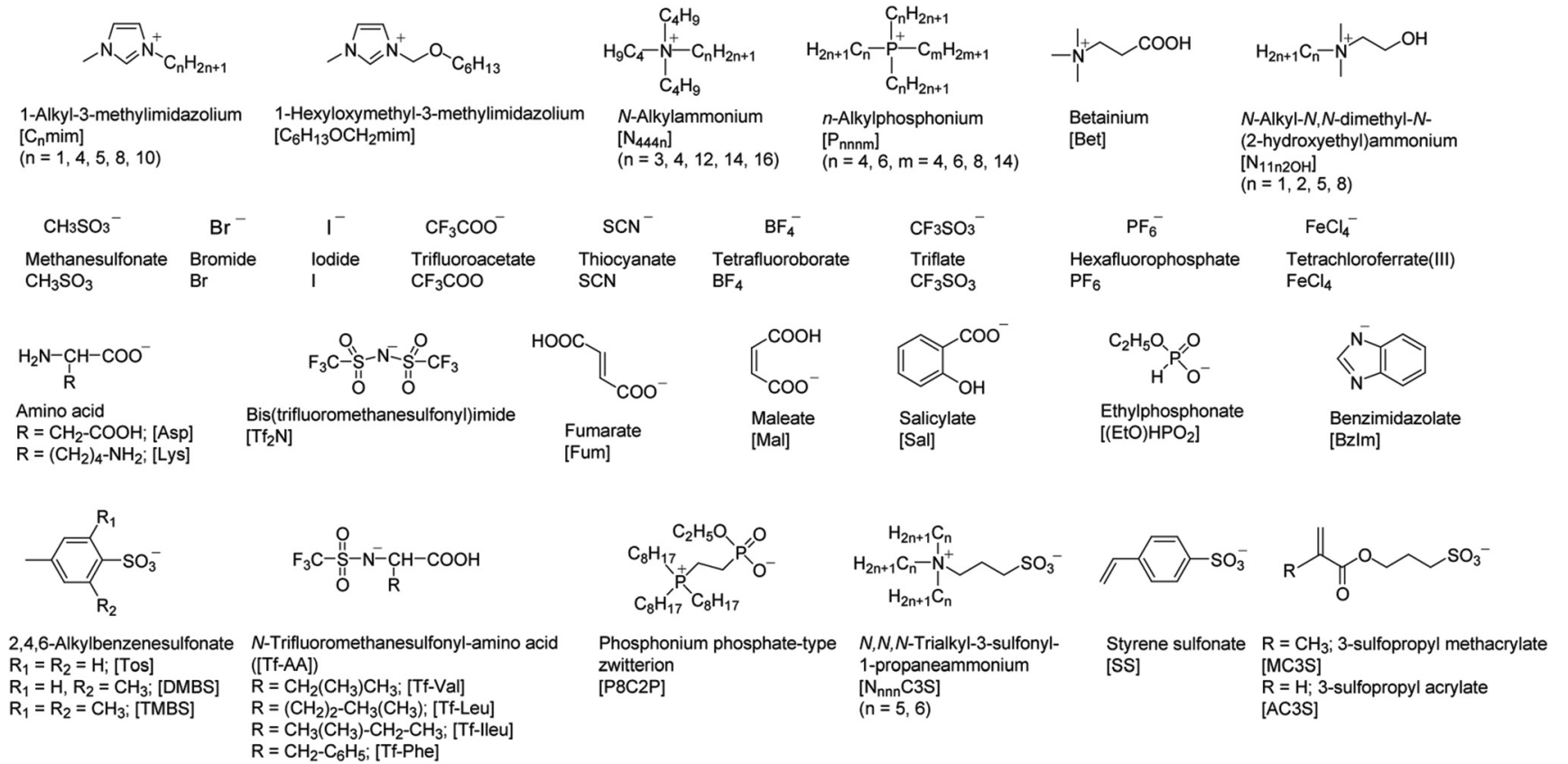

Fig. 1 Component ions of ILs used in this study.

$n$-alkylammonium salts were also reported at that time (entries 10, 11). ${ }^{41-43}$ Rebelo and co-workers reported ternary systems comprised of $\left[\mathrm{C}_{4} \mathrm{mim}\right] \mathrm{PF}_{6}$-water-ethanol showing the LCSTtype phase transition using ethanol as a co-solvent (entry 12). ${ }^{44}$ With respect to IL/water mixtures, the LCST-type phase transition was first observed in $N$-trifluoromethanesulfonylamino acid ([Tf-AA $]^{-}$)-based ILs with tetra- $n$-butylphosphonium cation reported by Ohno and co-worker (entry 13). ${ }^{45}$ The same group also reported that a $\left[\mathrm{P}_{4444}\right][\mathrm{Tf}-\mathrm{Leu}] /$ water mixture underwent a reversible phase change between a homogeneous phase and liquid/liquid biphases by bubbling $\mathrm{CO}_{2} / \mathrm{N}_{2}$ gasses, as well as a small temperature change. ${ }^{46}$ This interesting phase behaviour was driven by the dissociation change of the carboxylic acid group onto the [Tf-Leu] ${ }^{-}$anion upon a slight change of $\mathrm{pH}$ of the aqueous solution. ${ }^{46}$

Fig. 3 presents images showing highly temperature-sensitive LCST-type phase transition of the IL/water mixed system. An aqueous solution containing $35 \mathrm{wt} \%\left[\mathrm{P}_{4444}\right] \mathrm{CF}_{3} \mathrm{COO}$ (entry 16) was homogeneous at $29{ }^{\circ} \mathrm{C}$ (Fig. 3 , left), and the solution was clearly phase separated into an aqueous upper phase and an IL-rich bottom phase at $34{ }^{\circ} \mathrm{C}$ or above (Fig. 3, right). These separated liquid/liquid biphases became miscible again upon

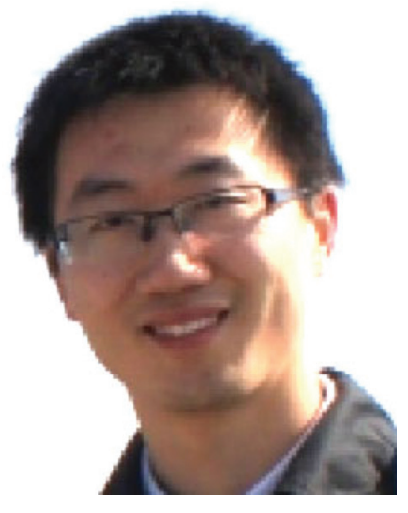

Yongjun Men
Dr Yongjun Men received his bachelor degree in Chemistry in Zhengzhou University, China in 2007, and his master degree in Polymer Chemistry in Beijing Normal University, China in 2011. Then, he moved to Max Planck Institute of Colloids and Interfaces, Germany, and completed his Ph.D. degree on a topic of poly(ionic liquid)-based thermoresponsive polymers and porous carbon materials under the supervision of Prof. Markus Antonietti and Dr Jiayin Yuan. At present, he is a postdoctoral researcher in the Department of Bio-organic Chemistry at Radboud University Nijmegen, the Netherlands. His current research focuses on the design of intelligent, self-propelled supramolecular assemblies as nano-engineered delivery systems.

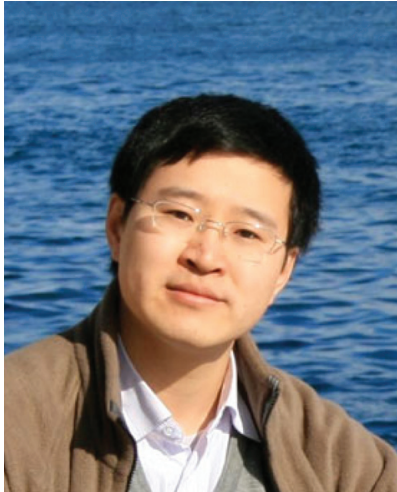

Jiayin Yuan
Dr Jiayin Yuan studied chemistry in Shanghai Jiao Tong University, China in 1998. He received his master degree in the University of Siegen, Germany in 2004. After his Ph.D. (summa cum laude) in the University of Bayreuth, Germany, under the supervision of Prof. Axel H. E. Müller in 2009, he joined the Department of Colloid Chemistry, directed by Prof. Markus Antonietti in the Max Planck Institute of Colloids and Interfaces in Potsdam, Germany. Since 2011, he has been a research group leader there. His current interest focuses on ionic liquid-based polymers and their materials applications. 

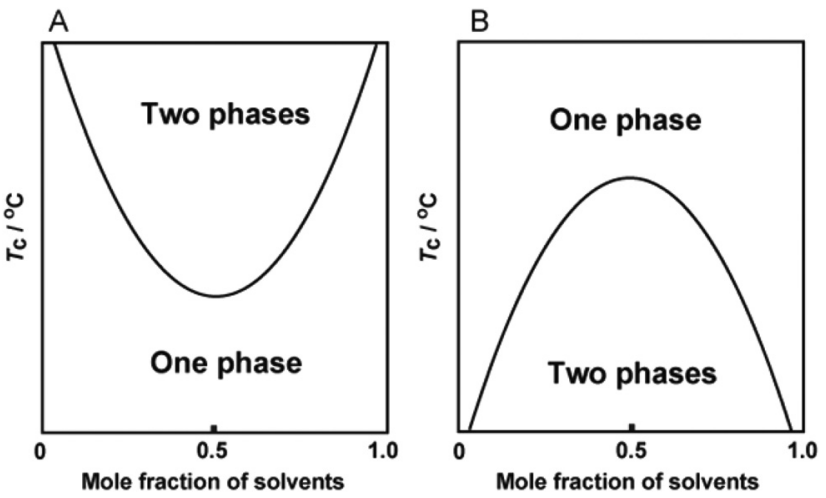

Fig. 2 Schematic illustration of typical phase diagrams of LCST behaviour (A) and UCST behaviour (B).

cooling it at $29{ }^{\circ} \mathrm{C}$ or below. This LCST-type phase change was found to be reversible and driven by a small temperature within $5^{\circ} \mathrm{C}$.

In terms of cationic structures, $n$-alkylphosphonium cations were commonly used to prepare ILs showing the LCST-type phase transition in water (entries 13, 14, 16, 17, 19-24, 26-28). ${ }^{33,45,46,48-52,54-56}$ The only exception is an iron(III)containing IL with $\left[\mathrm{C}_{4} \mathrm{mim}\right]^{+}$as a cation (entry 15$) .{ }^{47}$ Despite the structural analogy of cation structures, various kinds of anions have been combined with these $n$-alkylphosphonium cations to prepare ILs so far. Halogenate, carboxylate, sulfonate, phosphonate, amino acid, and benzimidazolate-based ILs exhibited the LCST-type phase transition with water by paring with adequately hydrophobic cations. The LCST-type phase transition was also found in aqueous zwitterion systems (entries 24, 25), ${ }^{52,53}$ in which both cation and anion are covalently tethered by an alkyl chain linker. One of the advanced properties of zwitterions is the fixation of the ion pair, and this will contribute to the distribution of ions in the media. Details will be reported elsewhere.

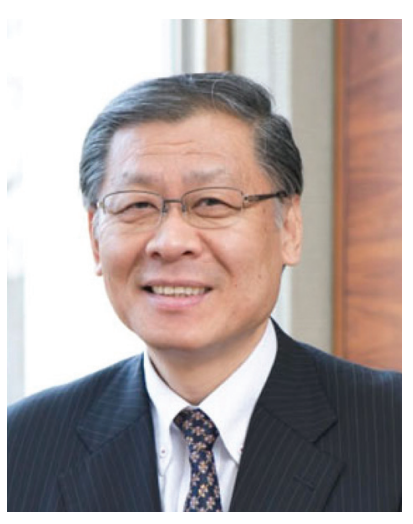

Hiroyuki Ohno
Professor Hiroyuki Ohno is known as one of leading scientists in ionic liquids. He received his Ph. D. degree from Waseda University in 1981. After working in Waseda University and Case Western Reserve University as a post-doctoral research fellow, he was invited to Tokyo University of Agriculture and Technology as an associate professor in 1988. In 1997, he was promoted to a full professor. He was elected as a vice dean of the graduate school in 2007 and then elected to be a dean from April, 2013. He has published 405 original papers in major journals and 170 reviews and books.
In addition to IL/water systems, the LCST behaviour of ILs with non-aqueous organic solvents is another characteristic thermoresponsive property. Seddon and co-workers reported mixed IL systems showing both UCST- and LCST-type phase transitions after mixing with chloroform (entry 29). ${ }^{57}$ Some other IL systems were reported to show the LCST-type phase transition with benzene derivatives (entries 30,31$){ }^{58,59}$ trifluoromethane under high pressures (entry 32 ), ${ }^{60}$ and several ether derivatives (entry 33). ${ }^{61}$ Yuan and co-workers reported $\left[\mathrm{C}_{1} \mathrm{mim}\right] \mathrm{I} / 1,4$-dipropoxypillar[5]arene/chloroform systems that undergo LCST-type phase transition via pillar[5]arene/IL host guest complexation (entry 34$)^{62}$

In aqueous IL systems, the $T_{\mathrm{c}}$ value is totally dependent on structures of component ions. With respect to the mixtures showing the UCST behaviour, the introduction of more hydrophobic ions increases the $T_{\mathrm{c}}$ value. ${ }^{28-30}$ On the other hand, the elevation of $T_{\mathrm{c}}$ values was generally found in the case of ILs composed of less hydrophobic ions, when the mixtures show the LCST behaviour. Upon comparison of several ILs with [Tf$\mathrm{AA}]^{-}$anions, the $T_{\mathrm{c}}$ value decreased according to the extension of alkyl chain length of side groups onto amino acids (entry 13). A similar trend was also observed in aqueous IL systems composed of [2,4,6-alkylbenzenesulfonate $]^{-}$anions (entry 17). Cationic structures also influence the $T_{\mathrm{c}}$ value. Similarly to anions, the $T_{\mathrm{c}}$ values decreased upon increasing the attached alkyl chains onto the phosphonium cations (entry 21, 23). Oppositely, a few ammonium-based salts with relatively longer alkyl groups onto the cations had higher $T_{\mathrm{c}}$ values upon increasing the number of alkyl chains (entry 11). In terms of center atom species of cations, phosphonium cations are effective to lower the $T_{\mathrm{c}}$ values than ammonium cations having the same alkyl chains (entries 17, 18). This is also comprehended to be due to the different hydrophobicity of ions. From the comparison of the $T_{\mathrm{c}}$ of ILs having phosphonium cations and those having ammonium cations, the increase in the hydrophobicity is empirically recognised to be comparable to the introduction of two methyl groups. ${ }^{48}$

It should be noted here that the added hydrophobicity/ hydrophilicity effect of both cations and anions governs the phase behaviour of the resulting ILs with water. ${ }^{63,64}$ The LCSTtype phase transition can be seen in the region between hydrophobic and hydrophilic ILs, when the phase behaviour of the IL/water mixtures were ordered according to the hydrophobicity of component ions. In other words, ILs comprised of moderately hydrophilic ions have potentials to show the LCST-type phase transition. To quantify the required range of hydrophobicity/hydrophilicity that allows for the LCST behaviour, the amount of dissolved water in the separated IL-rich phase from the aqueous phase was measured at $60{ }^{\circ} \mathrm{C}$, and converted into the number of water molecules per ion pair (hydrophilicity index; HI).$^{63}$ Upon considering a relationship between the phase behaviour and HI values, ILs with HI above 7 had a chance to undergo the LCST-type phase behaviour with water. Based on the required HI value for ILs, Ohno and co-workers successfully prepared a few IL monomers, in which a polymerisable vinyl group was introduced onto the component ions, 
Table 1 Thermoresponsive phase behaviour of ILs with solvents

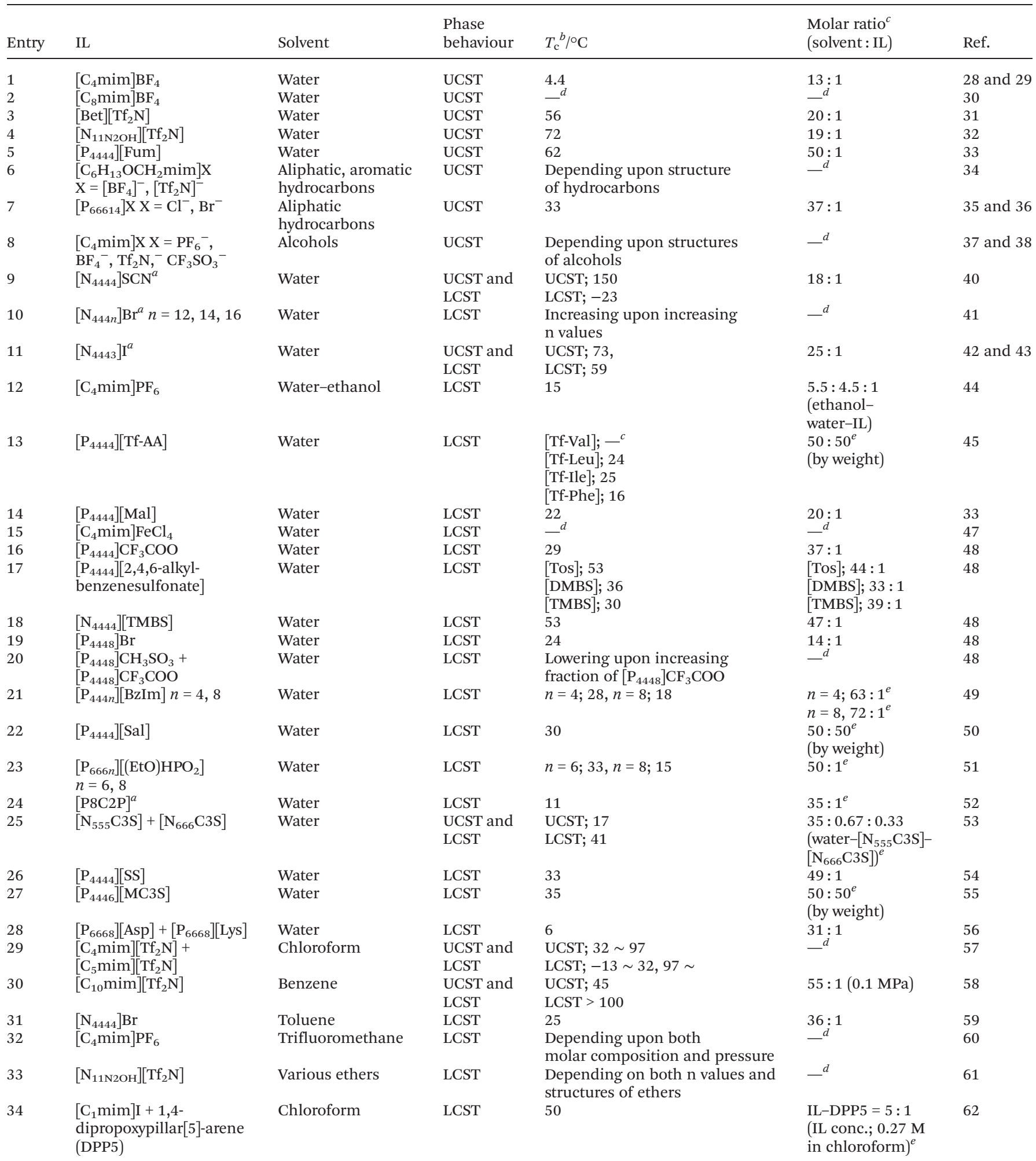

${ }^{a}$ Although these organic salts do not meet the definition of ILs, it is of significance to mention them here because of their structural analogy to ILs. ${ }^{b}$ Phase transition temperature. ${ }^{c}$ At critical composition. ${ }^{d}$ Not available. ${ }^{e}$ Critical composition was not observed, or not described. 


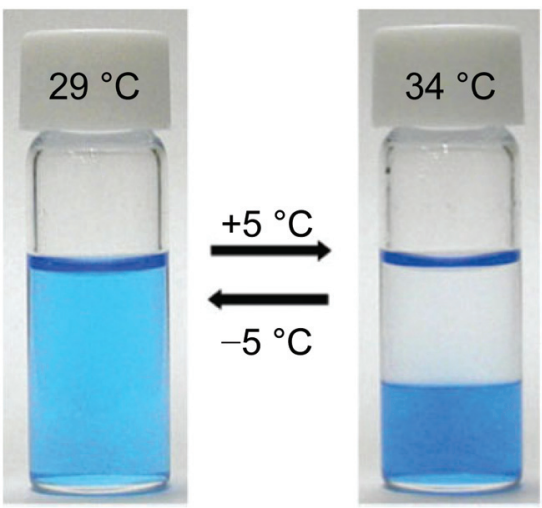

Fig. 3 Temperature-sensitive LCST-type phase transition of $\left[\mathrm{P}_{4444}\right]$ $\mathrm{CF}_{3} \mathrm{COO} /$ water mixture. ${ }^{48}$ The IL-rich phase was dyed using Coomassie Brilliant Blue.

showing the LCST-type phase transition with water by carefully choosing component ions (entries 26, 27). ${ }^{54,55}$ The detailed studies on the IL monomers and the resulting PILs are summarised in Chapter 3.

In general, the thermoresponsive IL/water mixtures can be obtained by careful design of the component ions to give an adequate hydrophobicity/hydrophilicity balance that allows for the LCST behaviour of the mixtures. Because the hydrophobicity/hydrophilicity balance is the most effective factor to control the LCST phase behaviour, mixing ILs is another practical method to realise the LCST phase behaviour of IL/water systems. Accordingly, if the target ILs are too hydrophilic to show the LCST-type phase transition, there should be still a chance to provide the thermoresponsive ILs by mixing moderately hydrophobic ILs. For example, a $\left[\mathrm{P}_{4448}\right] \mathrm{CH}_{3} \mathrm{SO}_{3} /\left[\mathrm{P}_{4448}\right]-$ $\mathrm{CF}_{3} \mathrm{COO}$ mixture is a couple of hydrophilic and hydrophobic ILs (entry 20). Although $\left[\mathrm{P}_{4448}\right] \mathrm{CH}_{3} \mathrm{SO}_{3}$ was miscible with water and $\left[\mathrm{P}_{4448}\right] \mathrm{CF}_{3} \mathrm{COO}$ was immiscible with water, the resulting mixtures turned to show the LCST behaviour. ${ }^{48}$ As the LCST phase behaviour should be the function of the total hydrophobicity/hydrophilicity balance, ${ }^{63}$ the IL mixture showed lower $T_{\mathrm{c}}$ value when the molar ratio of hydrophobic $\left[\mathrm{P}_{4448}\right]$ $\mathrm{CF}_{3} \mathrm{COO}$ increased. Based on the relation between the $T_{\mathrm{c}}$ values and total hydrophobicity of component ions, we can put various ions in the hydrophobicity order by measuring the $T_{\mathrm{c}}$ values after mixing target ions in the system that shows the LCST behaviour. ${ }^{65}$ Another interesting example for mixed IL systems is the mixtures of amino acid-based ILs (entry 28). ${ }^{56}$
Both $\left[\mathrm{P}_{6668}\right][$ Asp $]$ and $\left[\mathrm{P}_{6668}\right][$ Lys $]$ were miscible with water, but their mixtures started to show the LCST behaviour at a suitable mixing ratio. This unexpected behaviour was presumably derived from anion-anion interactions between the charged side chains onto the amino acids. ${ }^{56}$ This strongly suggested that the hydrated state of the component ions had profound effect on the phase behaviour of the IL/water mixtures.

\section{Thermoresponsive ionic liquid- derived homopolyelectrolytes}

It was strongly suggested that several physico-chemical properties of starting IL monomers, such as low glass transition temperature and inflammability should be maintained even after polymerisation. ${ }^{21,22}$ Accordingly, it is assumed that when IL monomers show LCST-type phase behaviour in water, the resulting polymers may also show the LCST behaviour. As a few IL monomers were newly found to show the LCST-type phase transition (e.g. $\left[\mathrm{P}_{4444}\right][\mathrm{SS}]$ and $\left.\left[\mathrm{P}_{4446}\right][\mathrm{MC} 3 \mathrm{~S}]\right)$, Ohno and co-workers polymerised the IL monomers and analysed the phase behaviour after mixing with water. Fig. 4 shows a typical synthetic procedure to obtain the PIL; poly $\left(\left[\mathrm{P}_{4444}\right][\mathrm{SS}]\right) .{ }^{54}$ The IL monomer was readily prepared via an anion exchange reaction between $\left[\mathrm{P}_{4444}\right] \mathrm{Cl}$ and sodium styrenesulfonate $(\mathrm{Na}[\mathrm{SS}])$. Then, free radical polymerisation was applied to $\left[\mathrm{P}_{4444}\right][\mathrm{SS}]$ using both $\alpha, \alpha^{\prime}$-azobis(isobutyronitrile) (AIBN) as a free radical initiator and water as a solvent. ${ }^{54}$ Because monomeric $\left[\mathrm{P}_{4444}\right]$ [SS] underwent the LCST-type phase transition with water and phase-separated upon heating, the reaction proceeded, in which, the monomer was suspended in the mixture. This simple polymerisation method was also examined for polymerisation of $\left[\mathrm{P}_{4446}\right][\mathrm{MC} 3 \mathrm{SS}] .^{55}$ Yuan and co-workers also prepared poly $\left(\left[\mathrm{P}_{4444}\right][\mathrm{SS}]\right)$ via a free radical polymerisation in ethanol as a solvent. ${ }^{66}$ In this case, the monomer was homogeneously dissolved in ethanol. The resulting polyelectrolytes as prepared via the suspended state ${ }^{54}$ or homogeneously dissolved state ${ }^{66}$ exhibited similar phase behaviour with water. The details are discussed below.

The resulting poly $\left(\left[\mathrm{P}_{4444}\right][\mathrm{SS}]\right)$ was found to undergo reversible LCST-type phase transition in water. Images in Fig. 4 show thermoresponsive phase behaviour of poly $\left(\left[\mathrm{P}_{4444}\right][\mathrm{SS}]\right) /$ water mixture in which $10 \mathrm{wt} \%$ polymer was dissolved in water. ${ }^{54}$ $\operatorname{Poly}\left(\left[\mathrm{P}_{4444}\right][\mathrm{SS}]\right)$ was miscible with water at $25{ }^{\circ} \mathrm{C}$, but the mixture became turbid upon heating at $57{ }^{\circ} \mathrm{C}$. This turbid

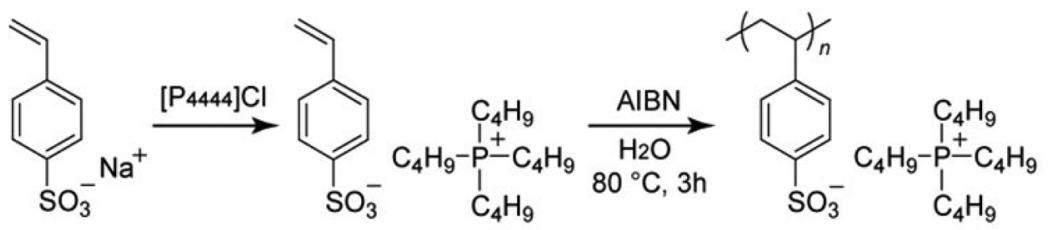

Fig. 4 Synthetic procedure of poly $\left(\left[\mathrm{P}_{4444}\right][\mathrm{SS}]\right)$ and its thermoresponsive behaviour with water.
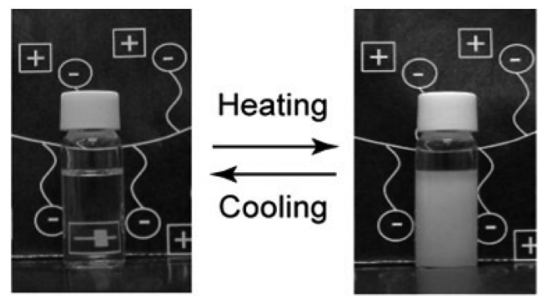
mixture turned clear again upon cooling. This type of thermoresponsive phase transition of PILs in pure water has never been reported. A number of non-ionic polymers have already been reported to show the LCST-type phase transition with water and other organic solvents. However, a wholly-charged homopolyelectrolyte that reversibly shows the LCST-type phase transition with water was not reported, especially, which was derived from IL monomers with a unique combination of physico-chemical properties of ILs. Generally, to prepare charged polymers of LCST-type phase transition in water, copolymerisation of a thermoresponsive non-ionic monomer with an ionic co-monomer was used. These studies are described in chapter 4.

Kohno and Ohno measured temperature dependence of turbidity of an aqueous solution containing $10 \mathrm{wt} \%$ poly $\left(\left[\mathrm{P}_{4444}\right]-\right.$ $[\mathrm{SS}]) .{ }^{54}$ On heating, the transmittance of the $\left[\mathrm{P}_{4444}\right][\mathrm{SS}] /$ water mixture dropped to $90 \%$ at $57{ }^{\circ} \mathrm{C}$ (Fig. 5, blue solid line). The transmittance of the solution sharply fell down upon further heating, and reached less than $1 \%$ at $59{ }^{\circ} \mathrm{C}$. The turbid solution became transparent again upon cooling (Fig. 5, blue dotted line). As shown in Fig. 5, there is a very small hysteresis on the solution turbidity. The reversible and sharp LCST behaviour of poly $\left(\left[\mathrm{P}_{4444}\right][\mathrm{SS}]\right)$ was also reported by both Yuan and co-workers ${ }^{66}$ and $\mathrm{Wu}$ and co-workers. ${ }^{67}$ Furthermore, the transition temperature $\left(T_{\mathrm{c}}\right)$ of poly $\left(\left[\mathrm{P}_{4444}\right][\mathrm{SS}]\right)$ was able to be readily decreased by copolymerising a little bit more hydrophobic monomer, such as $\left[\mathrm{P}_{4446}\right][\mathrm{SS}]$ (Fig. 5 , red line). ${ }^{54}$ As seen in Fig. 5 , introduction of $30 \mathrm{~mol} \%$ of $\left[\mathrm{P}_{4446}\right][\mathrm{SS}]$ in the copolymer made a shift of $T_{\mathrm{c}}$ to about $20^{\circ} \mathrm{C}$. The shift of the $T_{\mathrm{c}}$ should be the function of the copolymer composition. ${ }^{54}$ This behaviour was consistent with the properties of thermoresponsive non-polymerisable $\mathrm{IL} /$ water mixtures as mentioned above (i.e., mixing relatively hydrophobic ILs lowers the $T_{\mathrm{c}}$ values). Similar changes in $T_{\mathrm{c}}$ values were also observed in poly $\left(\left[\mathrm{P}_{4446}\right][\mathrm{MC} 3 \mathrm{~S}]\right)$ systems. ${ }^{68}$ Clearly, there is a close relation-

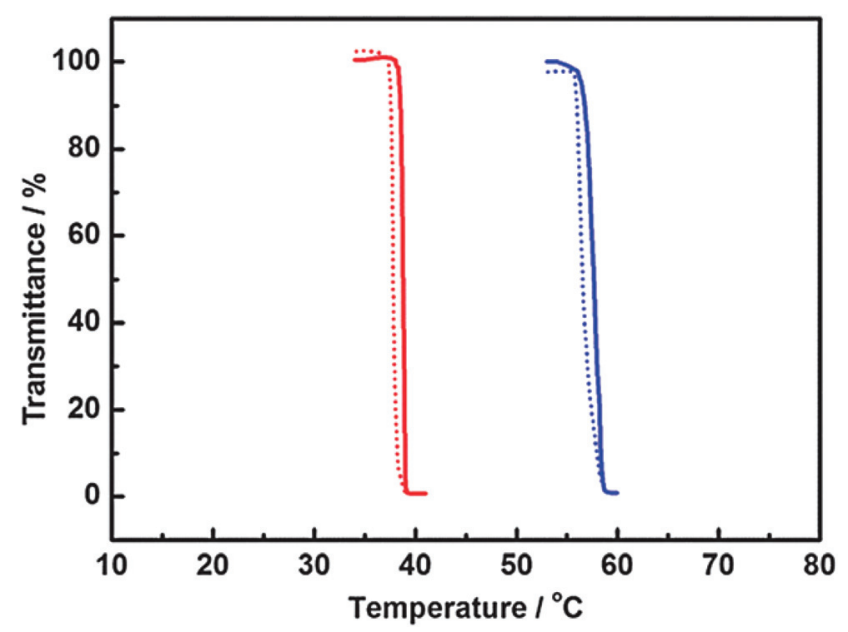

Fig. 5 Turbidity change of an aqueous solution containing $10 \mathrm{wt} \%$ poly $\left(\left[\mathrm{P}_{4444}\right][\mathrm{SS}]\right)$ (blue lines) or poly $\left(\left[\mathrm{P}_{4444}\right][\mathrm{SS}]_{0.7}-\mathrm{CO}-\left[\mathrm{P}_{4446}\right][\mathrm{SS}]_{0.3}\right.$ (red lines); molar ratio of $\left[\mathrm{P}_{4444}\right][\mathrm{SS}]$ to $\left[\mathrm{P}_{4446}\right][\mathrm{SS}]$ was 7.0 to 3.0$)$; solid lines: heating process, dotted lines: cooling process. ${ }^{54}$ ship between $\left[\mathrm{P}_{4444}\right][\mathrm{SS}]$ and poly $\left(\left[\mathrm{P}_{4444}\right][\mathrm{SS}]\right)$ in terms of the thermoresponsiveness. Based on these studies mentioned above, it is strongly suggested that designing monomeric IL systems to show the thermoresponsive phase behaviour is an essential strategy to prepare thermoresponsive PILs.

The aforementioned studies clearly demonstrate that the copolymerisation of IL monomers is a facile, potential, and accurate method to control the phase behaviour of polyelectrolytes in an aqueous solution. In the case of PNIPAM and other non-ionic polymers, copolymerisation with other comonomers having different hydrophobicity is also effective to control the phase behaviour. ${ }^{69}$ However, the phase response of the resulting copolymers often became slower than that of homopolymers upon a temperature change. ${ }^{70}$ To maintain the thermal sensitivity, efforts are being made to prepare the copolymers with very narrow molecular weight distribution. ${ }^{71}$ Against this, the reported PIL copolymers derived from two IL monomers retained very sharp phase-transition. This sharp temperature response of the PILs is one of the advantages for practical applications.

Although the UCST-type phase transition of PILs with various solvents has already been reported, the LCST-type phase transition of PIL/water mixtures was reported in the last two years. After the discovery of poly $\left(\left[\mathrm{P}_{4444}\right][\mathrm{SS}]\right)$, several other PIL homopolymers have been designed to show the LCST-type phase transition with water. We summarised the reported PIL homopolymers showing the LCST-type phase transition in water, along with other thermoresponsive PILs in water or organic solvents (Fig. 6 and Table 2). Fig. 6 presents structures of PILs showing thermoresponsive phase behaviour in solution. Ritter and co-workers presented "pseudo" LCST-type phase transition of hydrophobic alkylimidazolium-based PIL 1 with water via reversible complexation between $\left[\mathrm{Tf}_{2} \mathrm{~N}\right]^{-}$and cyclodextrins including randomly methylated $\beta$-cyclodextrin (RAMEB-CD). ${ }^{72}$ Ohno and co-workers reported anionic PIL homopolymers (PIL 2 and PIL 3) showing the LCST-type phase transition with water. ${ }^{54,55}$ Yuan and co-workers reported a phosphonium-based cationic PIL $4,^{73}$ and Ohno and coworkers reported ammonium-based cationic PIL 5 and 6 showing the LCST behaviour with water. ${ }^{74}$ Yuan and coworkers also found the LCST behaviour of gemini-type dicationic PIL 7 with water. ${ }^{75}$ Aoshima and co-workers prepared several types of alkylimidazolium-based cationic PILs (PIL 8 and 18) and alkylpyridinium-based cationic PIL 19 via living cationic polymerisation that show UCST- or LCST-type phase transition with water or a few organic solvents such as chloroform. ${ }^{76,77}$ Very recently, Luis and co-workers prepared amino acid-based chiral-PILs via bulk reversible addition-fragmentation chain-transfer (RAFT) polymerisation (PIL 20 and 23). ${ }^{78}$ These PILs underwent the LCST-type phase transition in chloroform stabilised by $2 \%$ of ethanol addition. ${ }^{78}$

Table 2 summarises both phase behaviour and $T_{\mathrm{c}}$ values of thermoresponsive PIL homopolymers, as well as polymerisation methods to obtain these polymers. Thermoresponsive turbidity measurement is a facile and effective method to determine the $T_{\mathrm{c}}$ values of the PIL/solvent mixtures. The $T_{\mathrm{c}}$ 
<smiles></smiles>

PIL 1<smiles>CCC(C)c1ccc([S+](=O)(=O)[O-])cc1</smiles>

PIL 2

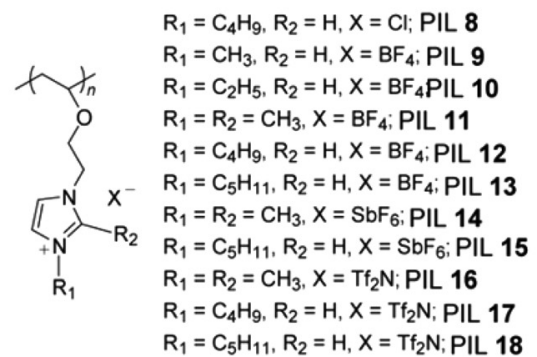

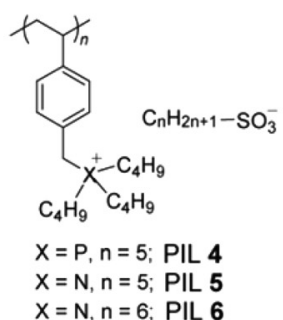

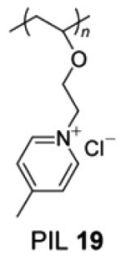

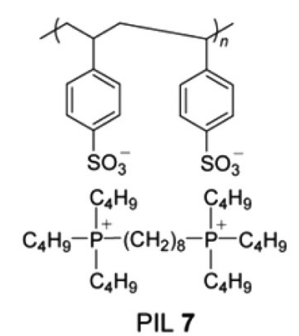

PIL 7

Fig. 6 Structures of thermoresponsive PIL homopolymers.

Table 2 Thermoresponsive behaviour of PIL homopolymers with solvents

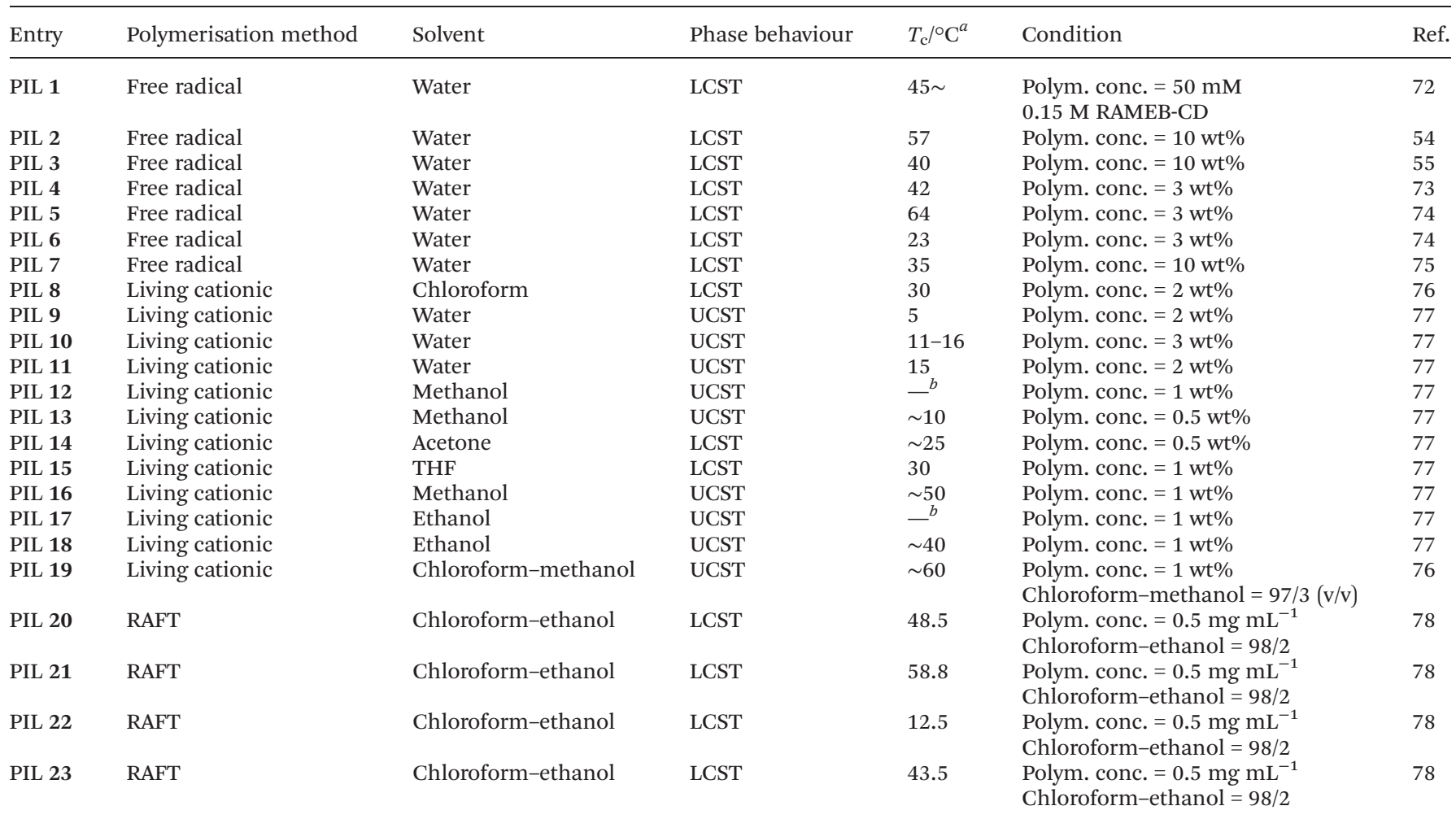

${ }^{a}$ The $T_{\mathrm{c}}$ values were determined by turbidity measurement. ${ }^{b}$ Data not shown.

values of PIL/solvent mixtures vary widely with structures of component ions, concentration of polymers, and solvent species. In the case of PIL/water mixtures showing the LCST behaviour, total hydrophobicity/hydrophilicity of component ions of IL monomers strongly affects the $T_{\mathrm{c}}$ values. This effect on the $T_{\mathrm{c}}$ values seems more noticeable than that for $\mathrm{IL} /$ water mixtures. For instance, upon comparing ammonium-based PIL 5 and phosphonium-based PIL $\mathbf{4}$ with the same number of alkyl chains, PIL
4 has a $T_{\mathrm{c}}$ value $42{ }^{\circ} \mathrm{C}$ lower than PIL $5 .^{74}$ The introduction of hydrophobic groups onto the component ions lowered the $T_{\mathrm{c}}$ values in thermoresponsive PIL/water systems. ${ }^{74}$ In PIL/chloroform systems, Luis and co-workers reported that the $T_{\mathrm{c}}$ value considerably dropped from 48.5 to $12.5{ }^{\circ} \mathrm{C}$ when another phenyl group was introduced into the cation (PIL 20 and 22). ${ }^{78}$

Polymer concentration also influences the $T_{\mathrm{c}}$ value. Despite the fact that the $T_{\mathrm{c}}$ values of some non-ionic polymers such as 
PNIPAM are almost independent from polymer concentration, thermoresponsive PILs have wide range of $T_{\mathrm{c}}$ values depending on polymer concentration. The LCST of PIL/water mixtures was higher upon decreasing the concentration..$^{55,66,73-75}$ The LCST of PIL/chloroform mixtures also became higher upon decreasing the concentration. ${ }^{78}$ On the other hand, the UCST of PIL/ water mixtures increases upon increasing the concentration. ${ }^{77}$ At highly diluted concentration, the resulting mixtures have no longer an optically detectable phase transition. ${ }^{73,74}$

In terms of the difference in $T_{\mathrm{c}}$ values between IL monomer and PIL, Ohno and co-workers reported that the $T_{\mathrm{c}}$ of $\left[\mathrm{P}_{4444}\right]$ $[\mathrm{SS}]$ and poly $\left(\left[\mathrm{P}_{4444}\right][\mathrm{SS}]\right)$ at the same concentration (10 $\mathrm{wt} \%$ in water) was 40 and $57^{\circ} \mathrm{C}$, respectively. ${ }^{54}$ They also reported that the $T_{\mathrm{c}}$ value of monomeric and polymerised $\left[\mathrm{P}_{4446}\right][\mathrm{C} 3 \mathrm{~S}]$ was 44 and $40{ }^{\circ} \mathrm{C}$ (10 wt $\%$ in water), respectively. ${ }^{55}$ This certainly pointed out that the $T_{\mathrm{c}}$ of this polymer should be a function of the average molecular weight of the PILs. Aoshima and coworkers analysed the effect of number-average molecular weight $\left(M_{\mathrm{n}}\right)$ on UCST of PIL $\mathbf{1 0}$ with narrow molecular weight distributions. ${ }^{77}$ Aqueous solutions of $3 \mathrm{wt} \%$ PIL 10 with a hydrophilic end group had $T_{\mathrm{c}}$ values at 11 and $16^{\circ} \mathrm{C}$, whereas PIL 11 with a hydrophobic end group had $T_{\mathrm{c}}$ values at 11 and $14^{\circ} \mathrm{C}$ regardless of the $M_{\mathrm{n}}$ values. These data demonstrated that the effect of molecular weight or the chain-end structure on the $T_{\mathrm{c}}$ values was small even in the low molecular weight region. ${ }^{77}$

In addition to the design of the component ions of IL monomers, addition of suitable salts was proved to be another effective method to control the $T_{\mathrm{c}}$ values of thermoresponsive PILs. Yuan and co-workers reported that the $T_{\mathrm{c}}$ value of poly$\left(\left[\mathrm{P}_{4444}\right][\mathrm{SS}]\right)$ increased dramatically upon adding $\mathrm{KBr}$ as a salt, but decreased via addition of $\left[\mathrm{P}_{4444}\right] \mathrm{Br}$ into the aqueous solution. ${ }^{66}$ Ohno and co-workers reported that the $T_{\mathrm{c}}$ value was much lower than that with pure water when phosphate buffer containing $\mathrm{KH}_{2} \mathrm{PO}_{4}-\mathrm{K}_{2} \mathrm{HPO}_{4}$ was used instead of pure water for poly $\left(\left[\mathrm{P}_{4446}\right][\mathrm{MC} 3 \mathrm{~S}]\right)$ systems. ${ }^{55}$ This opposite trend depending on salt species suggests that the kosmotropicity of salts, ${ }^{55,66}$ fraction of the salts in an aqueous phase and polymer phase, ${ }^{55}$ and/or ion exchange reaction ${ }^{75}$ strongly influence the $T_{\mathrm{c}}$ values.

Very recently, Ohno and co-workers reported a simple "blending" method to tune the LCST behaviour of PILs in water. ${ }^{79}$ The blending method allows to obtain thermoresponsive PILs whose LCSTs can be controlled by blending plural PILs with different hydrophobicity. In addition, a few mixtures of hydrophobic and hydrophilic PILs, both of which do not show the LCST-type phase behaviour, turned into thermoresponsive PILs capable of undergoing the LCST behaviour in water.

It should be a straightforward idea to fabricate various kinds of solid-state materials made from thermoresponsive PILs, as several PIL homopolymers have already been reported to show the LCST behaviour. Recently, a few types of crosslinked thermoresponsive PIL materials have been reported. Based on fundamental studies on LCST-type phase transition of PIL homopolymer/water systems by both Ohno's group and Yuan's group as mentioned above, Diamond and co-workers first prepared PIL-based hydrogels composed of $\left[\mathrm{P}_{4444}\right][\mathrm{SS}]$ and
$\left[\mathrm{P}_{4446}\right][\mathrm{AC} 3 \mathrm{~S}]$ as IL monomers. ${ }^{80}$ The hydrogels were prepared via photopolymerisation of the monomers using 2-hydroxy-2methylpropiophenone as a photoinitiator. Stable and transparent gels were obtained when sufficiently long cross-linkers were introduced into the monomers, such as polypropylene glycol diacrylate with $M_{\mathrm{n}}$ of 800 (PPO800) and polyethylene glycol diacrylate with $M_{\mathrm{n}}$ of 700. Ohno and co-workers also prepared PIL hydrogels using both $\left[\mathrm{P}_{4446}\right][\mathrm{MC} 3 \mathrm{~S}]$ and $\left[\mathrm{P}_{4448}\right]-$ [MC3S] as IL monomers and 1,14-tetradecanediol dimethacrylate as a cross-linker in the presence of a small amount of water $(20 \mathrm{wt} \%) .{ }^{68}$ The thermal polymerisation of the monomers was undertaken at $80{ }^{\circ} \mathrm{C}$ using AIBN as a radical initiator. In this case, the polymerisation was carried out in a homogeneously dissolved state. Yuan and co-workers reported gemini-type dicationic phosphonium-based hydrogels with $[\mathrm{SS}]^{-}$as an anion (PIL 7 in Table 2). ${ }^{75}$ In case of the dicationic monomer system, it was expected that a gel would be produced without any cross-linkers, because the polymer chains would be cross-linked by ionic interactions through the dicationic part. However, the resulting polymers did not become gel. Instead, the hydrogel could be prepared using divinylbenzene as a crosslinker, AIBN as a radical initiator, and relatively large amount of water as a solvent at $80^{\circ} \mathrm{C}$. At this high temperature and concentration, the monomer was phase-separated from water and the polymerisation proceeded under heterogeneous condition.

Diamond and co-worker studied the thermoresponsive shrinking behaviour of PIL-based hydrogels in which $5 \mathrm{wt} \%$ PPO800 was used as a cross-linker. ${ }^{80}$ Fig. 7 shows the normalised diameters of the hydrogels upon heating. These PIL gels shrunk gradually upon heating over a wide range of temperatures $\left(\sim 40{ }^{\circ} \mathrm{C}\right)$. Ohno and co-workers also reported that the prepared poly $\left(\left[\mathrm{P}_{4446}\right][\mathrm{MC} 3 \mathrm{~S}]\right)$-based hydrogels underwent desorption of water by elevating the temperature. ${ }^{68}$ The thermoresponsive behaviour of both systems clearly shows that the resulting hydrogels maintain the LCST-type phase behaviour with water. ${ }^{68,75,80}$ The $T_{\mathrm{c}}$ values of the thermoresponsive PIL hydrogels deeply depended on structure of both IL monomers

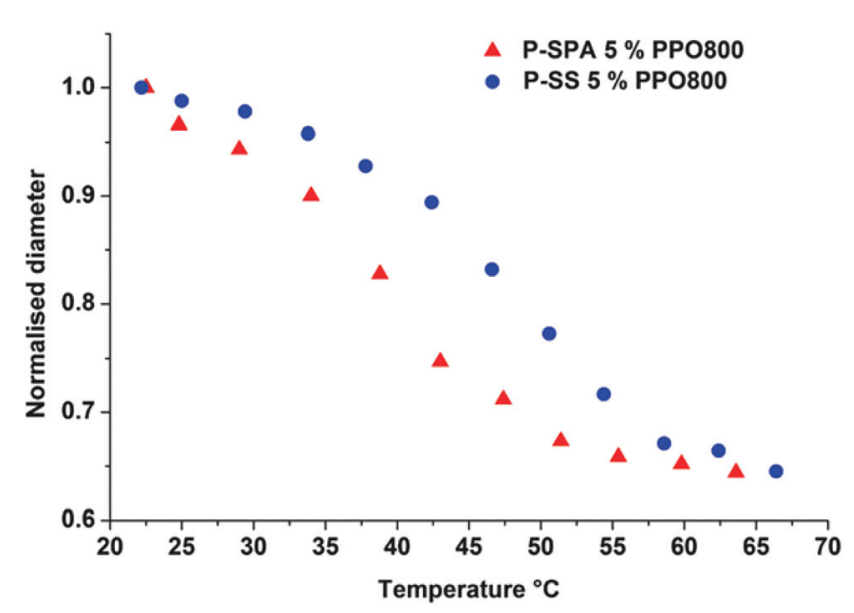

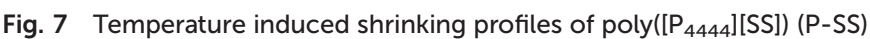
and poly $\left(\left[\mathrm{P}_{4446}\right][\mathrm{AC} 3 \mathrm{~S}]\right)$ (P-SPA) both cross-linked with $5 \mathrm{~mol} \%$ of $\mathrm{PPO}^{2} 00^{80}$ 
and cross-linkers. In spite of a very sharp phase transition in case of liner PILs, the cross-linked PIL-based hydrogels displayed a significant broadening of the thermoresponsiveness. ${ }^{68,80}$ This might be due to the inhomogeneity of the crosslinking density in the hydrogels ${ }^{68}$ and/or the decreased level of freedom of the bulky PIL in the gel. ${ }^{80} \mathrm{~A}$ broader endothermic peak of PIL hydrogels compared with that of linear PILs supported this suggestion. ${ }^{68,80}$ In addition, some of reported PIL hydrogels were still fragile, and its mechanical strength was not strong enough for reversible water absorption/ desorption. ${ }^{68}$ These poor mechanical properties will be solved in the near future.

Apart from thermoresponsive PIL/water systems, Wang and co-workers reported highly cross-linked PIL nanogels (CLNs) that show UCST behaviour in methanol. ${ }^{81}$ This system consists of gemini-type bisimidazolium monomer (1,4-butanediyl-3,3'bis-1-vinylimidazolium halides), ethylene glycol dimethacrylate, and AIBN. The resulting mixtures were polymerised in methanol to form CLNs, whose particle sizes totally depended on the ratio of the components in the range of less than $100 \mathrm{~nm}$. Interestingly, a few CLNs thus prepared underwent reversible sol-gel phase transition behaviour, and the solution converted to a gel state upon cooling. Such a simple method to prepare highly-temperature sensitive CLNs will be applied to many thermoresponsive PIL systems.
To provide an insight into the mechanism of thermoresponsive behaviour is another important subject emerging from a fundamental viewpoint. As several thermoresponsive PILs were readily available with relatively large quantities, only a few studies for the analysis of the LCST behaviour for both ILs and PILs have been published recently. Wu and co-workers performed two-dimensional infrared correlation spectroscopy (2D IR) in order to reveal the dynamic phase transition of both monomeric and polymerised $\left[\mathrm{P}_{4444}\right][\mathrm{SS}]$ after mixing with water. ${ }^{67}$ This measurement is useful in determining sequential order of different chemical groups by the analysis of both the synchronous and asynchronous spectra. ${ }^{82}$ A few typical vibration modes from 2D IR spectroscopy for monomeric $\left[\mathrm{P}_{4444}\right][\mathrm{SS}] /$ water mixtures (e.g. $\mathrm{CH}$ group of $\left[\mathrm{P}_{4444}\right]^{+}$cation and $\mathrm{SO}_{3}{ }^{-}$group of $[\mathrm{SS}]^{-}$anion) suggested that $\left[\mathrm{P}_{4444}\right]^{+}$cation started to aggregate, and eventually hydrogen bonds between water and $[\mathrm{SS}]^{-}$were broken by heating them up to nearly critical temperature (i.e., $\left.T_{\mathrm{c}}\right)$. Of poly $\left(\left[\mathrm{P}_{4444}\right][\mathrm{SS}]\right) /$ water mixtures, however, the $\mathrm{SO}_{3}{ }^{-}$group of $[\mathrm{SS}]^{-}$anion was primarily wrapped into the aggregated globules, subsequently $\left[\mathrm{P}_{4444}\right]^{+}$cation was distributed around the globules. Combined with other analytical methods including DSC, NMR, and FT-IR measurements, synergetic variations of both $\left[\mathrm{P}_{4444}\right]^{+}$cation and $[\mathrm{SS}]^{-}$anion of $\left[\mathrm{P}_{4444}\right][\mathrm{SS}]$ resulted in a randomly aggregated structure of globules during heating (Fig. 8, upper). In contrast, a repeating
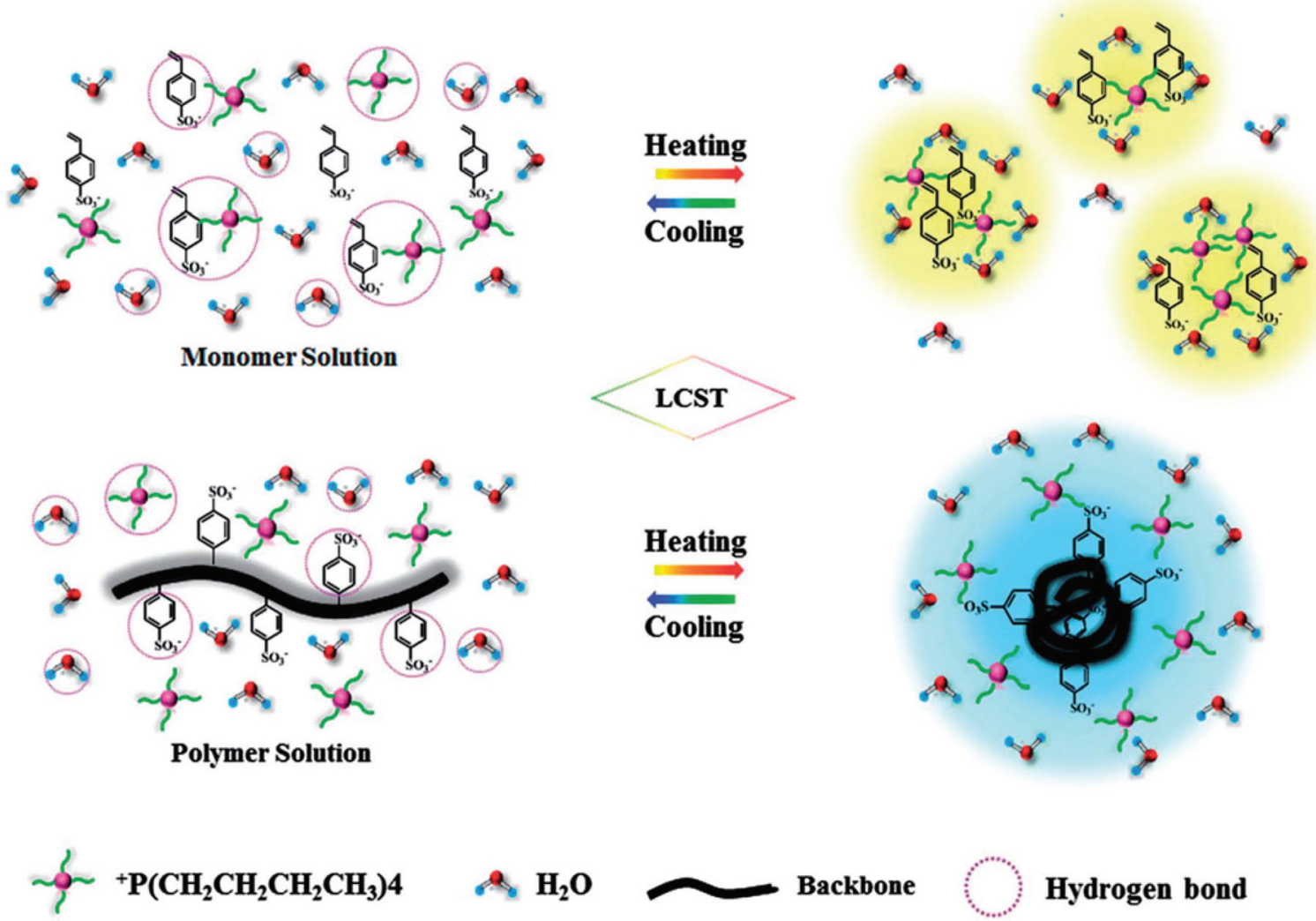

Backbone

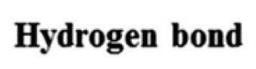

Fig. 8 Schematic illustration of the LCST-type phase transition of $\left[\mathrm{P}_{4444}\right][\mathrm{SS}]$ (upper) and poly $\left(\left[\mathrm{P}_{4444}\right][\mathrm{SS}]\right)$ (bottom) in water during the heating and cooling processes. ${ }^{67}$ 
unit of $[\mathrm{SS}]^{-}$anion in poly $\left[\mathrm{P}_{4444}\right][\mathrm{SS}]$ dominantly contributed to forming the aggregated globules. (Fig. 8, bottom).

The morphological change of thermoresponsive PILs was reported by Ohno and co-workers using polycationic type PILs. ${ }^{74}$ Combined with transmission electron microscopy (TEM), dynamic light scattering (DLS) and microscopy, PIL 4 (see Table 2) was found to form polydispersed nanospheres in water at a temperature below the $T_{\mathrm{c}}$ value. The nanospheres merged into micrometer-sized unimodal microspheres upon heating above the $T_{\mathrm{c}}$ value. ${ }^{74}$ Gao and co-workers also studied thermoresponsive phase behaviour of non-polymerisable $\left[\mathrm{P}_{4444}\right] \mathrm{CF}_{3} \mathrm{COO}$ with water by DLS, UV-vis spectroscopy, and freeze-fracture transmission electron microscopy (FF-TEM). ${ }^{83}$ Below the $T_{\mathrm{c}}$ value, $\left[\mathrm{P}_{4444}\right] \mathrm{CF}_{3} \mathrm{COO}$ formed long-living spherical aggregates in water without any surfactants, which was confirmed by FF-TEM. The size of aggregates enlarged upon elevating temperatures, and a remarkable increase of their size was observed at a temperature near the $T_{\mathrm{c}}$ value. Both reports from Ohno and co-workers and Gao and co-workers strongly suggest that the morphological transition of charged spherical aggregates in water at and around the $T_{\mathrm{c}}$ values features the thermoresponsive behaviour of both ILs and PILs. With respect to $\mathrm{IL} /$ water mixtures, further increase of temperature above the $T_{\mathrm{c}}$ value resulted in liquid/liquid biphases composed of hydrated IL phases and aqueous phases. ${ }^{48}$ On the other hand, hydrated gel-like phases were seen in a few PIL systems upon heating the mixture far above the $T_{\mathrm{c}^{\mathrm{c}}}{ }^{55}$ These primary reports will facilitate a lot of subsequent studies especially in the field of analytical chemistry. Indeed, multiple analyses will be required to comprehend the mechanism of the thermoresponsive phase behaviour of both IL/solvent systems and PIL/solvent systems, by exploiting different combination of cation/anion pairs.

\section{Thermoresponsive copolymers of poly(ionic liquid)s}

Other than PIL homopolymers presenting thermoresponsive behaviour, the copolymers of an IL monomer and a non-ionic monomer are also interesting to study. The copolymer not only adds another running factor but also further broadens the property and functional window of the thermal responsive systems. From the standpoint of copolymer structure, there are in principal two types of thermoresponsive PIL copolymers, i.e., block-type and random copolymers of IL monomers with non-charged conventional monomers. Considering the copolymer composition, the copolymer can be constructed from a thermoresponsive PIL and a non-thermoresponsive neutral polymer, or vice versa. Before the discovery of thermoresponsive polyelectrolyte homopolymers, a normal way was to build up the copolymers from a non-thermoresponsive PIL and a traditional thermoresponsive neutral polymer, like PNIPAM, which presents an LCST-type phase behaviour around $32{ }^{\circ} \mathrm{C}$ in an aqueous solution, above which the polymer experiences a coil-to-globule transition. Here PILs are expected to influence the physical properties of PNIPAM and its surrounding environment, leading to a change of $T_{\mathrm{c}}$.

Yuan's group synthesised a random copolymer via free radical copolymerisation of NIPAM and 1-ethyl-3-vinylimidazolium bromide (Fig. 9, PIL-PNIPAM 1) ${ }^{84}$ The LCST of the copolymer strongly depended on the molar ratio of the IL to non-ionic unit, and its $T_{\mathrm{c}}$ value could be precisely tuned by the addition of external salts. Due to the strong cation- $\pi$ interaction between the imidazolium cation and carbon nanotube wall, the copolymer can serve as a "smart" waterborne dispersion of carbon nanotubes that respond to both temperature and ionic strength in the solution. One apparent advantage of this system is the ability to design the precipitation event of the well-dispersed carbon nanotubes in aqueous solution at any liquid water temperature range from room temperature up to $100{ }^{\circ} \mathrm{C}$. Based on these experiments, a theoretical model was constructed to explain the salt effect and as a guide for future design of thermoresponsive materials. $^{85}$

To attain well-defined copolymer nanostructures via selfassembly in an aqueous solution, block copolymer is frequently used, especially double-stimuli-responsive diblock copolymers for self-assembly of core-shell nanoparticles. Texter and co-workers employed a facile route to synthesise PIL diblock copolymer (Fig. 9, PIL-PNIPAM 2) by a radical chain polymerisation. ${ }^{86}$ By setting the chain extension reaction at different temperatures, two lengths, i.e., 60 and $250 \mathrm{kDa}$, of the PIL block, poly(1-(11-acryloylundecyl)-3-methyl imidazolium bromide) [poly(ILBr), were prepared using 2,2'-azobis[2methyl- $N$-(2-hydroxyethyl)propionamide] as a radical initiator. Then the PNIPAM block grew from the PIL chain end, specifically the $\alpha$-methylene group adjacent to the hydroxyl group, via a redox initiator comprising $\mathrm{Ce}^{4+}$, which eventually produced a PIL- $b$-PNIPAM diblock copolymer. Elevating the temperature above $32{ }^{\circ} \mathrm{C}$ (i.e., the LCST of PNIPAM), collapses the PNIPAM block and leads to a colloid dispersion with particles of 1-2 $\mu \mathrm{m}$ in diameter. The particles were generated from the diblock copolymer, i.e., PNIPAM block condensed as a core and a stretched PIL block as a shell to stabilise the particle in water. On the contrary, when at room temperature a defined amount of $\mathrm{KBr}$ salt was added into the block copolymer solution, the PIL chain block collapsed to form a dense core, and the PNIPAM block acted as corona to solubilise the core-shell nanoparticles. The total size of the particles is in a range of $300-400 \mathrm{~nm}$. In another report, by grafting the same poly(ILBr) from both ends of a telechelic poly(propylene oxide) macroinitiator via an atom transfer radical polymerisation, a highly water-soluble triblock poly(ILBr)-b-PPO- $b$-poly(ILBr) (Fig. 9, PIL-PO 1) was synthesised by Texter and co-workers. ${ }^{87}$ This triblock copolymer showed thermoresponsive sol-gel transition when a weight fraction of the polymer in water was above 0.33 . The gel phase continues with increasing temperature, even persisting to more than $80^{\circ} \mathrm{C}$. The thermoresponsive morphological changes of these block copolymers, as mentioned above, would expand the possibility of various PILs as thermoresponsive materials. 


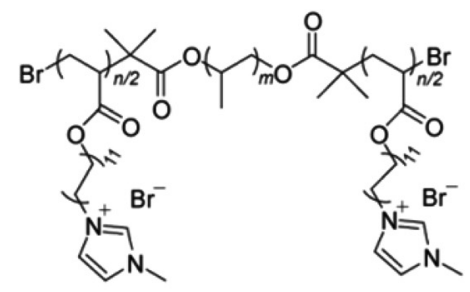

PIL-PO 1

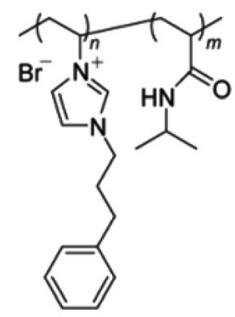

PIL-PNIPAM 3

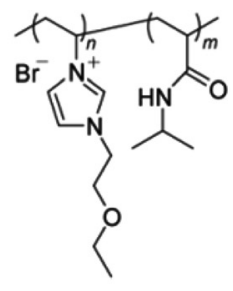

PIL-PNIPAM 4<smiles>CC[n+]1ccn(C(C)(C)CCCCC(C)C(=O)NC(C)C)c1</smiles>

PIL-PNIPAM 1

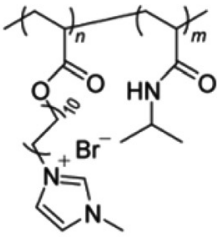

PIL-PNIPAM 2

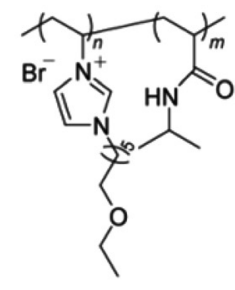

PIL-PNIPAM 5

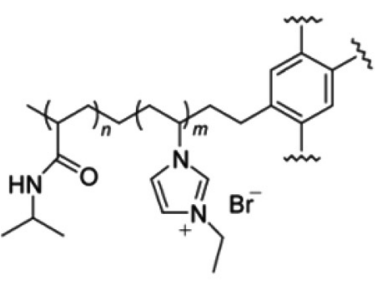

PIL-PNIPAM 7

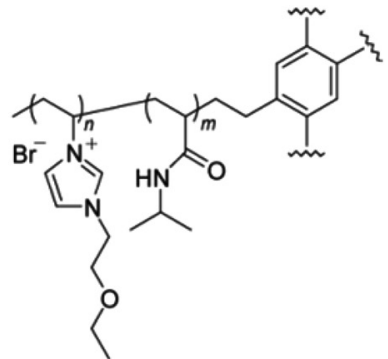

PIL-PNIPAM 6

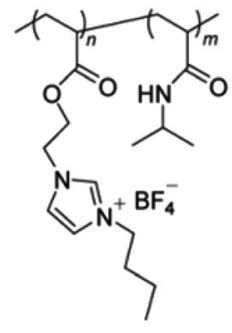

PIL-PNIPAM 8<smiles></smiles>

PIL-PNIPAM 9

Fig. 9 PIL-based copolymers showing LCST behaviour after mixing with water.

To accurately control the length of the diblock copolymer, RAFT, using xanthate compounds, was employed to synthesise three types of PIL- $b$-PNIPAM copolymers (Fig. 9, PIL-PNIPAM 3-5) with relatively low polydispersity by Mori and coworkers. $^{88}$ When the PIL block was more hydrophobic, the LCST of the copolymer in the absence of salt would be lower than the critical temperature of pure PNIPAM. By using these block copolymers, the assembly formed colloid particles with a diameter around $166 \mathrm{~nm}$, depending on the block length of each polymer chain. When counter anion was changed from the hydrophilic bromide to hydrophobic tetrafluoroborate, the PIL block turned less miscible with water, thus they formed micelles even at room temperature. Furthermore, the same group synthesised two types of star block copolymers containing a PIL segment and PNIPAM as a thermoresponsive segment (Fig. 9, PIL-PNIPAM 6, 7). ${ }^{89}$ A PIL segment can be arranged either as an outer block of the arm or as an inner part. At temperatures above the LCST of the PNIPAM segment, the star block copolymers consisting of PINPAM as the outer block showed a two-step transition process, from small micelle $(\sim 200 \mathrm{~nm})$ to large dehydrated clusters $(600-700 \mathrm{~nm})$. In contrast, when PINPAM was designed as the inner part, the copolymer underwent a rapid aggregation process, and the unimers dissolved in water were transformed into a large micellar cluster. Tenhu's group synthesised a block copolymer from a PIL containing a tetrafluoroborate anion and PNIPAM (Fig. 9. PIL-PNIPAM 8) by RAFT polymerisation with different length of PNIPAM. ${ }^{90}$ They observed that the block copolymer showed no endotherms when the ratio of PIL to PNIPAM was below 1, i.e., with a short PNIPAM block the phase transition upon heating was very weak and did not lead to the formation of large aggregates. However, increasing the length of PNIPAM to 2-4 times longer than the PIL block, the polymers should aggregate into micelles with a turbidity transition at $30-37^{\circ} \mathrm{C}$.

PIL copolymer (nano) particles obtained from the blockcopolymer self-assembly are not structurally as stable as the particles prepared by traditional heterophase polymerisation, such as emulsion polymerisation. Yuan and co-workers synthesised PIL nanoparticles via aqueous dispersion polymerisation of a vinyl imidazolium-based IL monomer, bearing a long alkyl chain with a distal ATRP initiating group (Fig. 9, PIL-PNIPAM 9). ${ }^{91}$ The PIL nanoparticles are extremely stable in water, and the sizes can be tuned from 25 to $120 \mathrm{~nm}$ by polymerisation at different monomer concentrations. PNIPAM brushes were then grafted from the surface of PIL nanoparticles to form a shell via ATRP, which shows responsiveness to temperature and ionic strength in an aqueous solution. More PIL colloid systems are summarised in another review paper. ${ }^{92}$ 
It should be noted that to date, no block copolymers constructed from a thermoresponsive PIL block and a non-ionic block have been studied. Furthermore, an ionic homopolymer may turn thermoresponsive by addition of a specific salt, as reported by Tenhu's group very recently. ${ }^{93}$ Because in this case the ion exchange occurs between the polymer and the added salt, the polymer backbone is in fact paired with two different types of counter ions, i.e., it exists in a "virtual copolymer" state. Related to this report, a polyelectrolyte copolymer composed of two non-thermoresponsive IL (or ionic) monomers may be possibly thermoresponsive even without salt addition. Such a fine control of the thermoresponsive behaviour of nonthermoresponsive PILs was carried out in a few PIL systems by copolymerising suitable IL monomers or blending PILs with different hydrophobicity. ${ }^{79}$

\section{Potential (expected) applications}

In spite of infancy of this field, given a unique combination of physico-chemical properties of ILs and thermoresponsiveness, various kinds of applications can be expected by exploiting thermoresponsive PILs, as well as thermoresponsive IL systems. In the case of thermoresponsive IL/solvent systems, several potential applications have already been proposed, for example, as liquid state materials. Some research groups have demonstrated the effectiveness of this specific application. Because some IL/solvent systems show reversible phase change between the homogeneous phase and liquid/liquid biphasic phases at ambient temperature change, both Ohno's group and Binnemans's group independently established energy-saving extraction systems that separate various compounds (e.g. biopolymers ${ }^{94,95}$ and metal ions ${ }^{96}$ ) from the aqueous phase to IL-rich phase. Koo and co-workers used the LCST-type phase transition of IL/water mixtures as reaction/ product separation media for enzymatic hydrolysis of penicil- lin. ${ }^{97}$ These studies eminently inspire the potential applications of thermoresponsive PILs as solid state materials. Yuan and co-workers already applied thermoresponsive PILs as dispersion/accumulation agents for carbon materials ${ }^{66}$ and gold nanoparticles. ${ }^{73}$ Luis and co-workers reported thermoresponsive PIL-modified gold nanoparticles, which show the LCSTtype phase transition in chloroform. ${ }^{78}$ Ohno and co-workers proposed the utilisation of thermoresponsive PIL hydrogels for potential desalination applications. ${ }^{68}$

In addition to conventional applications of thermoresponsive non-ionic polymers (e.g. material carriers, actuators, separations, or sensing), one can conceive several applications for thermoresponsive PILs, especially in the fields of stabilisation, accumulation, extraction, and separation of charged molecules. Fig. 10 summarises expected applications of thermoresponsive PILs as various solid-state materials (i.e., membranes, polymer grafted interfaces, nanoparticles, and hydrogels). Because these solid-state materials have already been fabricated from IL monomers with different structures, it should not be difficult to prepare these materials by using thermoresponsive IL monomers. The thermoresponsive PILbased membranes or grafted interfaces would be useful for constructing novel separation systems for several compounds including biopolymers (e.g. proteins, enzymes, and/or polysaccharides), because some ILs have an ability to dissolve these molecules selectively (Fig. 10, upper left and right). Binding/ release of target molecules in water would be another possible application for thermoresponsive PIL-based nanoparticles (Fig. 10, bottom left). Considering the high charge density of PILs, there would be no chance for other ions to dissolve in the PIL phase from the aqueous phase. Accordingly, the thermoresponsive PIL-based hydrogels would be applicable for desalination processes that reversibly absorb/desorb water molecules from salt water by a slight temperature change (Fig. 10, bottom right). These studies will be reported elsewhere in the near future.
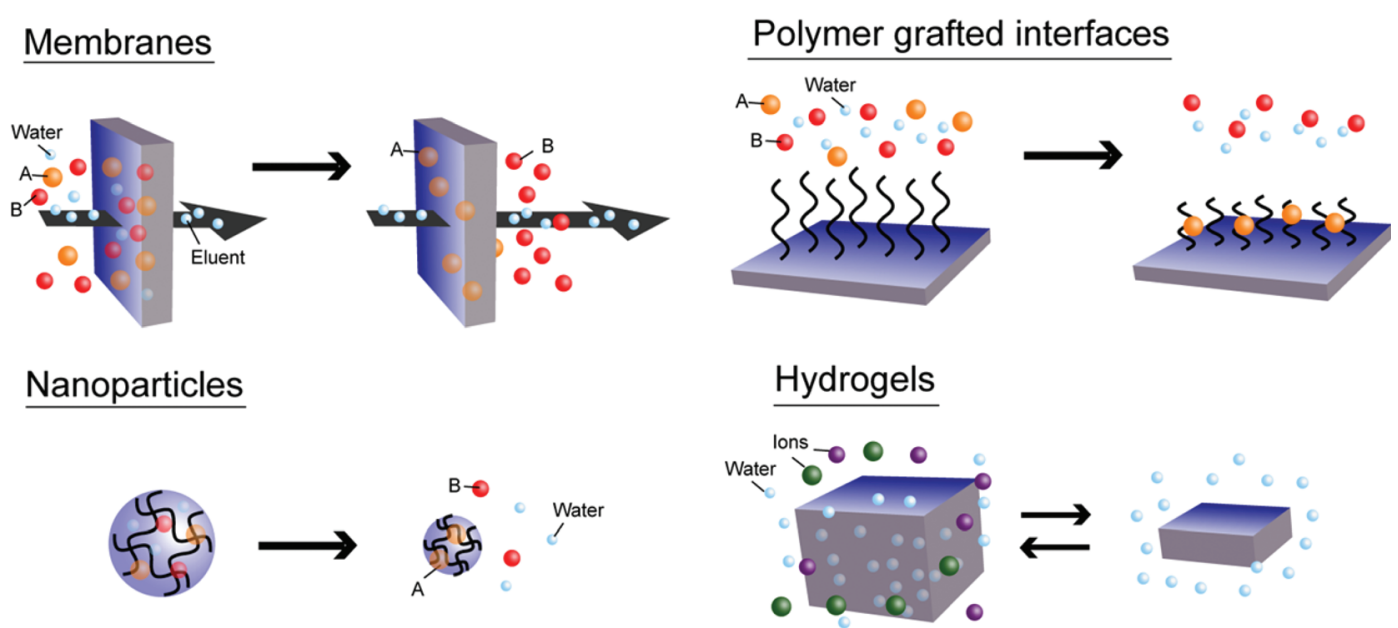

Fig. 10 Expected applications of thermoresponsive PILs as solid-state materials for separation/stabilisation of target molecules (as indicated "A" or "B" in the figure). 


\section{Conclusions and future outlook}

Recent development in the design of thermoresponsive PILS has been summarised in this review paper. The origin of their thermal responsiveness was inherently derived from the thermoresponsive phase behaviour of the IL/solvent interaction. These relationships between ILs and PILs were thoroughly described. Nevertheless, we anticipate that there are still many candidate PIL materials that have potential to show the thermoresponsive phase behaviour, upon considering the fact that various kinds of ILs were reported to undergo thermoresponsive behaviour with many solvents so far. Given the various physico-chemical properties depending on the structural diversity of the component ions, these thermoresponsive PIL systems provide the opportunity for discovering unprecedented phenomena and applications not previously realised for these classical neutral thermoresponsive polymers.

\section{Acknowledgements}

This study was supported by a Grant-in-Aid for Scientific Research from the Japan Society for the Promotion of Science (no. 26248049). Y.K. acknowledges the Japan Society for the Promotion of Sciences (JSPS) for financial support (Postdoctoral Fellowship for Research Abroad). S.S. acknowledges JSPS for financial support (Research Fellowship for Young Scientists). J.Y. thanks the Max Planck Society for the financial support.

\section{Notes and references}

1 T. Tanaka, Phys. Rev. Lett., 1978, 40, 820-823.

2 H. G. Schild, Prog. Polym. Sci., 1992, 17, 163-249.

3 M. Shibayama, T. Norisuye and S. Nomura, Macromolecules, 1996, 29, 8746-8750.

4 J.-F. Lutz, Ö. Akdemir and A. Hoth, J. Am. Chem. Soc., 2006, 128, 13046-13047.

5 H. Nuhn and H.-A. Klok, Biomacromolecules, 2008, 9, 27552783.

6 L. Ayres, M. R. J. Vos, P. J. H. M. Adams, I. O. Shklyarevskiy and J. C. M. van Hest, Macromolecules, 2003, 36, 5967-5973.

7 J. C. Rodriguez-Cabello, J. Reguera, A. Girotti, F. J. Arias and M. Alonso, Adv. Polym. Sci., 2006, 200, 119-167.

8 D. L. Nettles, A. Chilkoti and L. A. Setton, Adv. Drug Delivery Rev., 2010, 62, 1479-1485.

9 Y. Lu, A. Wittemann, M. Ballauff and M. Drechsler, Macromol. Rapid Commun., 2006, 27, 1137-1141.

10 D. Schmaljohann, Adv. Drug Delivery Rev., 2006, 58, 16551670.

11 T. Okano, N. Yamada, M. Okuhara, H. Sakai and Y. Sakurai, Biomaterials, 1995, 16, 297-303.

12 M. Ebara, M. Yamato, T. Aoyagi, A. Kikuchi, K. Sakai and T. Okano, Biomacromolecules, 2004, 5, 505-510.
13 E. S. Gil and S. M. Hudson, Prog. Polym. Sci., 2004, 29, 1173-1222.

14 A. M. Schmidt, Colloid Polym. Sci., 2007, 285, 953-966.

15 A. Kikuchi and T. Okano, Prog. Polym. Sci., 2002, 27, 11651193.

16 F. A. Plamper, A. Schmalz, M. Ballauff and A. H. E. Müller, J. Am. Chem. Soc., 2007, 129, 14538-14539.

17 F. A. Plamper, M. Ruppel, A. Schmalz, O. Borisov, M. Ballauff and A. H. E. Müller, Macromolecules, 2007, 40, 8361-8366.

18 T. Welton, Chem. Rev., 1999, 99, 2071-2083.

19 N. V. Plechkova and K. R. Seddon, Chem. Soc. Rev., 2008, 37, 123-150.

20 P. Wasserscheid and T. Welton, Ionic Liquids in Synthesis, Wiley-VCH, Weinheim, 2nd edn, 2008.

21 K. Ito and H. Ohno, Chem. Lett., 1998, 27, 751-752.

22 H. Ohno, Electrochim. Acta, 2001, 46, 1407-14111.

23 J. Lu, F. Yan and J. Texter, Prog. Polym. Sci., 2009, 34, 431448.

24 D. Mecerreyes, Prog. Polym. Sci., 2011, 36, 1629-1648.

25 J. Yuan, D. Mecerreyes and M. Antonietti, Prog. Polym. Sci., 2013, 38, 1009-1036.

26 N. Nishimura and H. Ohno, Polymer, 2014, 55, 3289-3297.

27 T. Ueki, Polym. J., 2014, 46, 646-655.

28 J. E. L. Dullius, P. A. Z. Suarez, S. Einloft, R. F. de Souza and J. Dupont, Organometallics, 1998, 17, 815-819.

29 C. A. Cerdeiriña, J. Troncoso, C. P. Ramos, L. Romani, V. Najdanovic-Visak, H. J. R. Guedes, J. M. S. S. Esperança, Z. P. Visak, M. Nunes da Ponte and L. P. N. Rebelo, in Ionic Liquids III A: Fundamentals, Progress, Challenges, and Opportunities, ed. R. Rogers and K. R. Seddon, ACS Symp. Ser., 2005, ch. 13, vol. 901, pp. 175-186.

30 P. J. Dyson, D. J. Ellis and T. Welton, Can. J. Chem., 2001, 79, 705-708.

31 P. Nockemann, B. Thijs, S. Pittois, J. Thoen, C. Glorieux, K. V. Hecke, L. V. Meervelt, B. Kirchner and K. Binnemans, J. Phys. Chem. B, 2006, 110, 20978-20992.

32 P. Nockemann, K. Binnemans, B. Thijs, T. N. Parac-Vogt, K. Merz, A.-V. Mudring, P. C. Menon, R. N. Rajesh, G. Cordoyiannis, J. Thoen, J. Leys and C. Glorieux, J. Phys. Chem. B, 2009, 113, 1429-1437.

33 Y. Fukaya, K. Sekikawa, K. Murata, N. Nakamura and H. Ohno, Chem. Commun., 2007, 3089-3091.

34 U. Domańska and A. Marciniak, J. Chem. Thermodyn., 2005, 37, 577-585.

35 D. Saracsan, C. Rybarsch and W. Schröer, Z. Phys. Chem., 2006, 220, 1417-1437.

36 K. Anderson, H. Rodríguez and K. R. Seddon, Green Chem., 2009, 11, 780-784.

37 J. M. Crosthwaite, S. N. V. K. Aki, E. J. Maginn and J. F. Brennecke, J. Phys. Chem. B, 2004, 108, 51135119.

38 J. M. Crosthwaite, M. J. Muldoon, S. N. V. K. Aki, E. J. Maginn and J. F. Brennecke, J. Phys. Chem. B, 2006, 110, 9354-9361. 
39 A. Arce, M. J. Earle, S. P. Katdare, H. Rodríguez and K. R. Seddon, Chem. Commun., 2006, 2548-2550.

40 H. Glasbrenner and H. Weingärtner, J. Phys. Chem., 1989, 93, 3378-3379.

41 S. A. Buckingham, C. J. Garvey and G. G. Warr, J. Phys. Chem., 1993, 97, 10236-10244.

42 H. Weingärtner, M. Kleemeier, S. Wiegand and W. Schröer, J. Stat. Phys., 1995, 78, 169-196.

43 M. Kleemeier, W. Schröer and H. Weingärtner, J. Mol. Liq., 1997, 73-74, 501-511.

44 V. Najdanovic-Visak, J. M. S. S. Esperança, L. P. N. Rebelo, M. N. da Ponte, H. J. R. Guedes, K. R. Seddon and J. Szydłowski, Phys. Chem. Chem. Phys, 2002, 4, 1701-1703.

45 K. Fukumoto and H. Ohno, Angew. Chem., Int. Ed., 2007, 46, 1852-1855.

46 Y. Kohno, H. Arai and H. Ohno, Chem. Commun., 2011, 47, 4772-4774.

47 Z.-L. Xie and A. Taubert, ChemPhysChem, 2011, 12, 364368.

48 Y. Kohno, H. Arai, S. Saita and H. Ohno, Aust. J. Chem., 2011, 64, 1560-1567.

49 Y. Tsuji and H. Ohno, Chem. Lett., 2013, 42, 527-529.

50 T. Ando, Y. Kohno, N. Nakamura and H. Ohno, Chem. Commun., 2013, 49, 10248-10250.

51 Y. Fukaya and H. Ohno, Phys. Chem. Chem. Phys., 2013, 15, 4066-4072.

52 Y. Fukaya and H. Ohno, Phys. Chem. Chem. Phys., 2013, 15, 14941-14944.

53 S. Saita, Y. Mieno, Y. Kohno and H. Ohno, Chem. Commun., 2014, 50, 15450-15452.

54 Y. Kohno and H. Ohno, Aust. J. Chem., 2012, 65, 91-94.

55 Y. Kohno, Y. Deguchi and H. Ohno, Chem. Commun., 2012, 48, 11883-11885.

56 S. Saita, Y. Kohno, N. Nakamura and H. Ohno, Chem. Commun., 2013, 49, 8988-8990.

57 J. Lachwa, J. Szydłowski, V. Najdanovic-Visak, L. P. N. Rebelo, K. R. Seddon, M. Nunes da Ponte, J. M. S. S. Esperança and H. J. R. Guedes, J. Am. Chem. Soc., 2005, 127, 6542-6543.

58 J. Łachwa, J. Szydłowski, A. Makowska, K. R. Seddon, J. M. S. S. Esperança, H. J. R. Guedes and L. P. N. Rebelo, Green Chem., 2006, 8, 262-267.

59 H. R. Dittmar and W. H. Schröer, J. Phys. Chem. B, 2009, 113, 1249-1252.

60 A. Yokozeki and M. B. Shiflett, AIChE J., 2006, 52, 39523957.

61 A. J. L. Costa, M. R. C. Soromenho, K. Shimizu, J. M. S. S. Esperança, J. N. C. Lopes and L. P. N. Rebelo, RSC Adv., 2013, 3, 10262-10271.

62 S. Dong, B. Zhen, Y. Yao, C. Han, J. Yuan, M. Antonietti and F. Huang, Adv. Mater., 2013, 25, 6864-6867.

63 Y. Kohno and H. Ohno, Phys. Chem. Chem. Phys., 2012, 14, 5063-5070.

64 Y. Kohno and H. Ohno, Chem. Commun., 2012, 48, 71197130.
65 S. Saita, Y. Kohno and H. Ohno, Chem. Commun., 2013, 49, 93-95.

66 Y. Men, X.-H. Li, M. Antonietti and J. Yuan, Polym. Chem., 2012, 3, 871-873.

67 W. Li and P. Wu, Polym. Chem., 2014, 5, 5578-5590.

68 Y. Deguchi, Y. Kohno and H. Ohno, Aust. J. Chem., 2014, 67, 1666-1670.

69 Y. G. Takei, T. Aoki, K. Sanui, N. Ogata, T. Okano and Y. Sakurai, Bioconjugate Chem., 1993, 4, 341-346.

70 T. Nishio, R. Suzuki, Y. Tsukada, H. Kanazawa, T. Okano and T. Miyabe-Nishiwaki, J. Chromatogr., A, 2009, 1216, 7427-7432.

71 S. Aoshima, S. Sugihara, M. Shibayama and S. Kanaoka, Macromol. Symp., 2004, 215, 151-163.

72 S. Amajjahe and H. Ritter, Macromolecules, 2008, 41, 32503253.

73 Y. Men, H. Schlaad and J. Yuan, ACS Macro Lett., 2013, 2, 456-459.

74 Y. Kohno, Y. Deguchi, N. Inoue and H. Ohno, Aust. J. Chem., 2013, 66, 1393-1398.

75 Y. Men, H. Schlaad, A. Voelkel and J. Yuan, Polym. Chem., 2014, 5, 3719-3724.

76 K. Seno, S. Kanaoka and S. Aoshima, J. Polym. Sci., Part A: Polym. Chem., 2008, 46, 5724-5733.

77 H. Yoshimitsu, A. Kanazawa, S. Kanaoka and S. Aoshima, Macromolecules, 2012, 45, 9427-9434.

78 S. Montolio, L. Gonzáez, B. Altava, H. Tenhu, M. Isabel Burguete, E. García-Verdugo and S. V. Luis, Chem. Commun., 2014, 50, 10683-10686.

79 Y. Deguchi, Y. Kohno and H. Ohno, Chem. Lett., DOI: 10.1246/cl.141016.

80 B. Ziółkowski and D. Diamond, Chem. Commun., 2013, 49, 10308-10310.

81 Y. Xiong, J. Liu, Y. Wang, H. Wang and R. Wang, Angew. Chem., Int. Ed., 2012, 51, 9114-9118.

82 I. Noda, J. Mol. Struct., 2008, 883, 2-26.

83 R. Wang, W. Leng, Y. Gao and L. Yu, RSC Adv., 2014, 4, 14055-14062.

84 S. Soll, M. Antonietti and J. Yuan, ACS Macro Lett., 2011, 1, 84-87.

85 J. Heyda, S. Soll, J. Yuan and J. Dzubiella, Macromolecules, 2014, 47, 2096-2102.

86 K. Tauer, N. Weber and J. Texter, Chem. Commun., 2009, 6065-6067.

87 J. Texter, V. A. Vasantha, C. R. rombez, R. Maniglia, L. Slater and T. Mourey, Macromol. Rapid Commun., 2012, 33, 69-74.

88 H. Mori, M. Yahagi and T. Endo, Macromolecules, 2009, 42, 8082-8092.

89 H. Mori, Y. Ebina, R. Kambara and K. Nakabayashi, Polym. J., 2012, 44, 550-560.

90 E. Karjalainen, N. Chenna, P. Laurinmaki, S. J. Butcher and H. Tenhu, Polym. Chem., 2013, 4, 1014-1024.

91 Y. Men, M. Drechsler and J. Yuan, Macromol. Rapid Commun., 2013, 34, 1721-1727. 
92 Y. Men, D. Kuzmicz and J. Yuan, Curr. Opin. Colloid Interface Sci., 2014, 19, 76-83.

93 E. Karjalainen, V. Aseyev and H. Tenhu, Macromolecules, 2014, 47, 7581-7587.

94 Y. Kohno, S. Saita, K. Murata, N. Nakamura and H. Ohno, Polym. Chem., 2011, 2, 862-867.
95 Y. Kohno, N. Nakamura and H. Ohno, Aust. J. Chem., 2012, 65, 1548-1553.

96 T. V. Hoogerstraete, B. Onghena and K. Binnemans, J. Phys. Chem. Lett., 2013, 4, 1659-1663.

97 N. L. Mai and Y.-M. Koo, Enzyme Microb. Technol., 2014, 63, 24-38. 